\title{
Technology development for ultraintense all-OPCPA systems
}

\author{
J. Bromage, S.-W. Bahk, I. A. Begishev, C. Dorrer, M. J. Guardalben, B. N. Hoffman, J. B. Oliver, \\ R. G. Roides, E. M. Schiesser, M. J. Shoup III, M. Spilatro, B. Webb, D. Weiner, and J. D. Zuegel \\ Laboratory for Laser Energetics, University of Rochester, Rochester, NY 14623-1299, USA \\ (Received 6 August 2018; revised 6 October 2018; accepted 13 November 2018)
}

\begin{abstract}
Optical parametric chirped-pulse amplification (OPCPA) [Dubietis et al., Opt. Commun. 88, 437 (1992)] implemented by multikilojoule Nd:glass pump lasers is a promising approach to produce ultraintense pulses $\left(>10^{23} \mathrm{~W} / \mathrm{cm}^{2}\right)$. Technologies are being developed to upgrade the OMEGA EP Laser System with the goal to pump an optical parametric amplifier line (EP OPAL) with two of the OMEGA EP beamlines. The resulting ultraintense pulses (1.5 kJ, $20 \mathrm{fs}$, $10^{24} \mathrm{~W} / \mathrm{cm}^{2}$ ) would be used jointly with picosecond and nanosecond pulses produced by the other two beamlines. A midscale OPAL pumped by the Multi-Terawatt (MTW) laser is being constructed to produce 7.5-J, 15-fs pulses and demonstrate scalable technologies suitable for the upgrade. MTW OPAL will share a target area with the MTW laser ( $50 \mathrm{~J}, 1$ to $100 \mathrm{ps}$ ), enabling several joint-shot configurations. We report on the status of the MTW OPAL system, and the technology development required for this class of all-OPCPA laser system for ultraintense pulses.
\end{abstract}

Keywords: nonlinear optics; optical parametric chirped-pulse amplification; ultrafast lasers; ultraintense lasers

\section{Introduction}

Ultraintense laser systems using the full potential of deuterated potassium dihydrogen phosphate (DKDP) for high-energy optical parametric chirped-pulse amplification (OPCPA) are being developed by a number of institutions $^{[1-3]}$. Noncollinear optical parametric amplifiers (NOPA's) comprised of DKDP produce broadband gain for supporting pulses as short as 10 fs centered near $920 \mathrm{~nm}$. Large-aperture DKDP crystals (>400 mm) make it possible to use Nd:glass lasers as kilojoule pump sources $^{[2]}$. Although OPCPA is now routinely used as a broadband front-end technology for many hybrid systems, scaling OPCPA to energies $>100 \mathrm{~J}$ is still an active area of laser research and development. This paper reports on a technology development program at the Laboratory for Laser Energetics where progress is being made toward the longterm goal of a femtosecond-kilojoule system pumped by the OMEGA EP laser ${ }^{[4]}$ by building a midscale all-OPCPA laser that is designed to address the technical challenges of the full-scale system.

Correspondence to: J. Bromage, Laboratory for Laser Energetics, University of Rochester, 250 E. River Rd., Rochester, NY 14623-1299, USA. Email: jbro@1le.rochester.edu

\section{EP OPAL concept}

\subsection{System overview}

In order to define areas of laser development necessary to achieve a full-scale ultraintense laser system, a preconceptual design was completed for adding a fifth beamline, known as an optical parametric amplifier line (EP OPAL), in the OMEGA EP Laser Bay to deliver $\sim 20-\mathrm{fs}, 1-\mathrm{kJ}$ laser pulses with focused intensities exceeding $10^{23} \mathrm{~W} / \mathrm{cm}^{2}$. The new beamline could be shot jointly with the existing kilojoule-nanosecond and kilojoule-picosecond OMEGA EP beams for experimental configurations not available elsewhere. The following sections give an overview of the main components of the system design and the technical challenges.

The concept shown in Figure 1 uses an ultrabroadband front end, a fifth NOPA stage (NOPA5), and two of the OMEGA EP beamlines to pump NOPA6 and NOPA7 for generating kilojoule pulses. Achromatic, all-reflective telescopes (not shown) image relay the ultrabroadband beam through these amplifier stages and into a four-grating compressor. The pulses are compressed to $20 \mathrm{fs}$ in a vacuum chamber and then transported and delivered to the targets in the OMEGA EP target chamber using a novel, two-stage focusing system. Powers and intensities for two $f$ numbers 


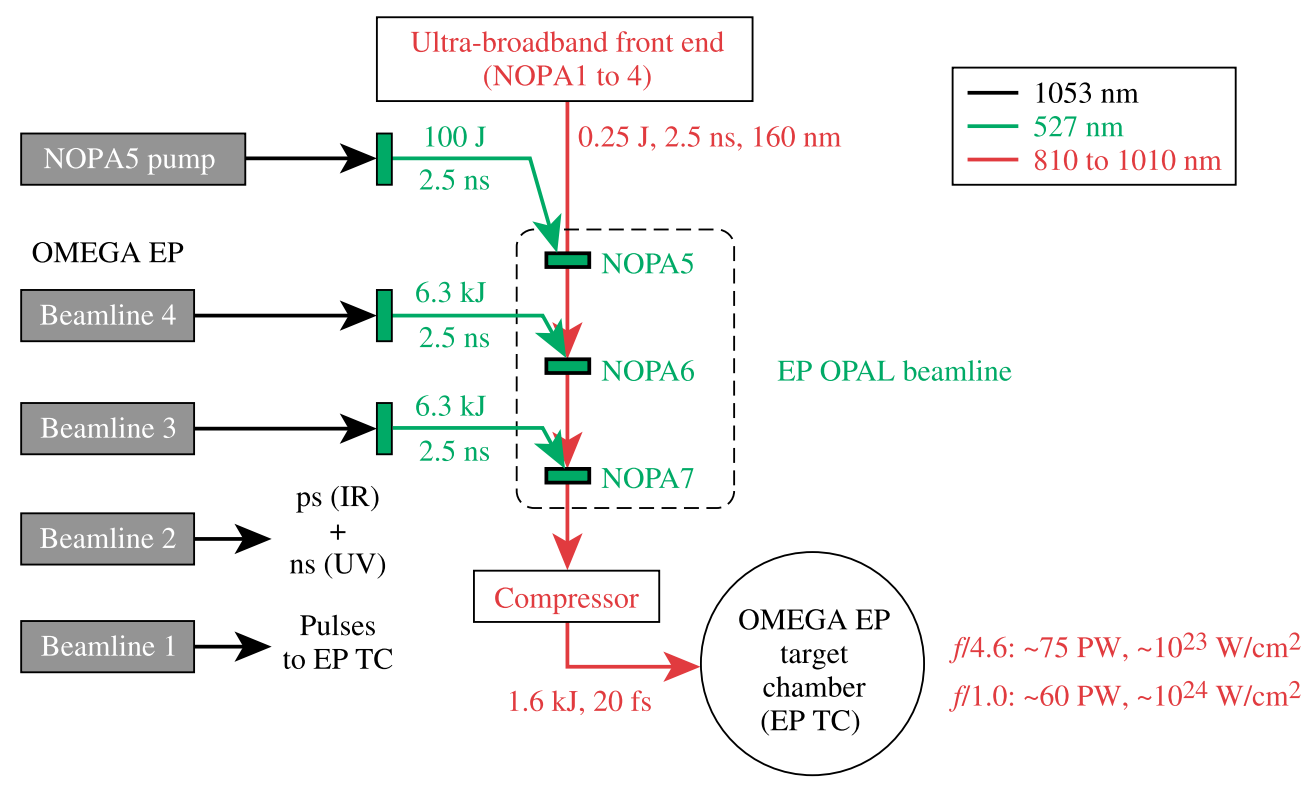

E23393J1

Figure 1. Top level of the EP OPAL (optical parametric amplifier line) system, showing the major subsystems and the neighboring OMEGA EP beamlines that would be available for joint shots. NOPA, noncollinear optical parametric amplifier; EPTC, OMEGA EP target chamber.

have been estimated. For joint shots, each of the Beamlines 1 and 2 can be an IR (picosecond) or UV (nanosecond) beam.

Figure 2 shows an isometric view of a preliminary layout for the EP OPAL architecture that uses the space reserved along the east side of the Laser Bay. The ultrabroadband front end is located directly below in one of the OMEGA EP Capacitor Bays. The broadband beam is transported through the Laser Bay floor to NOPA5, which is located at the south end of the EP OPAL. A new pump laser (2.5 ns, $526 \mathrm{~nm}, 100 \mathrm{~J}$ ) would be added to pump this amplifier. The NOPA5 output is image relayed with magnification to NOPA6 and then on to NOPA7. These amplifiers are pumped using the frequency-doubled output of Beamlines 4 and 3, respectively. The output of NOPA7 is image relayed to a vacuum compressor chamber, similar to the one used for OMEGA EP, where a four-grating compressor is used to produce $20 \mathrm{fs}$ pulses. The optics for transporting the beam to the target chamber are in vacuum, including a two-stage focusing system with an ellipsoidal plasma mirror as the final optic ${ }^{[5]}$.

\subsection{Technical challenges and critical technologies}

The EP OPAL conceptual design depends on resolving a range of technical challenges to achieve peak powers approaching $100 \mathrm{PW}$ and focused intensities up to $10^{24} \mathrm{~W} / \mathrm{cm}^{2}$. The main challenges identified in the preconceptual design, ranked in order of decreasing difficulty are as follows.

(1) Advanced gratings: Large-scale gratings that meet the bandwidth, damage threshold, and wavefront requirements are needed.
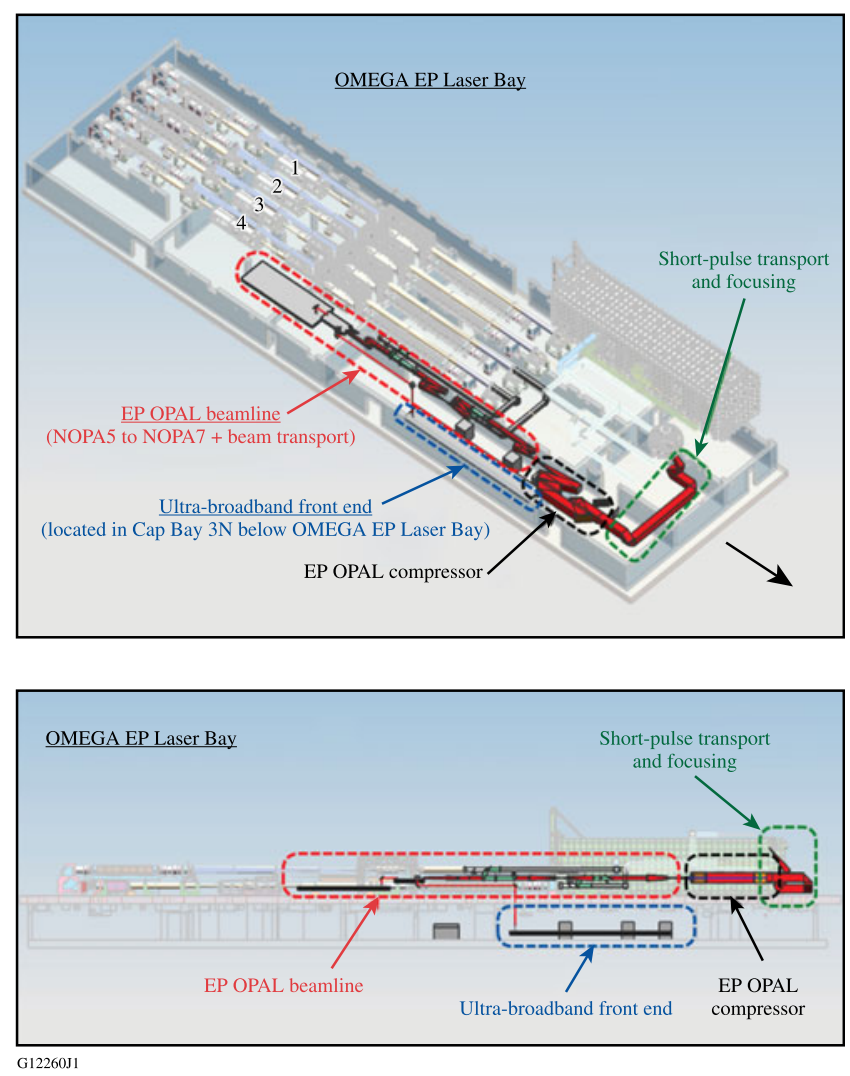

Figure 2. Isometric CAD views of the OMEGA EP Laser System, showing the locations of the main components of the EP OPAL system.

(2) Ultrabroadband wavefront control and focusing: Adaptive optical systems for wavefront correction and 


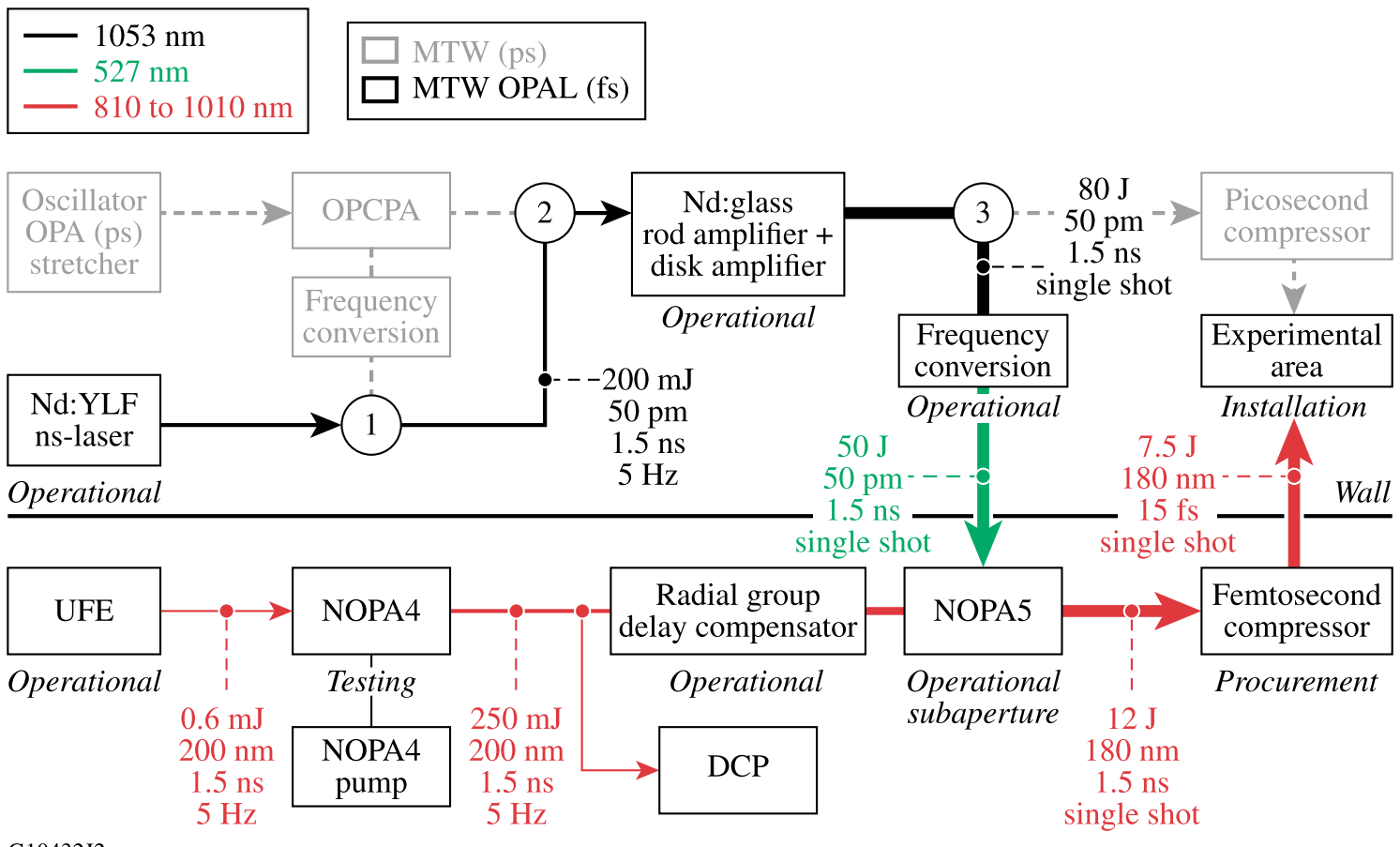

Figure 3. The Multi-Terawatt (MTW) OPAL system in relation to the existing MTW laser. The MTW laser is reconfigured using three switchyards. Portions of the MTW laser that are not used for pumping the final amplifier stage of OPAL (NOPA5) are grayed out. OPA, optical parametric amplifier; UFE, ultrabroadband front end; DCP, diagnostic compressor package.

two-stage focusing to achieve sub-5- $\mu \mathrm{m}$ focal spot are needed.

(3) Large-aperture, highly deuterated DKDP: OMEGA EP-scale crystals $(\sim 400 \mathrm{~mm})$ cut for Type-I phase matching with deuteration levels $>90 \%$ and high optical quality are needed.

(4) Short-pulse coatings: Scale coating prototypes developed for the Multi-Terawatt (MTW) OPAL beam size $(9 \mathrm{~cm})$ up to the EP OPAL beam size $(80 \mathrm{~cm})$, which requires laser optics and gratings significantly larger than those used on OMEGA EP (40-cm beams).

(5) Short-pulse diagnostics: Develop techniques to obtain a representative sample of the full-energy beam, and diagnostics for configuring the system before the shot.

(6) Ultrabroadband dispersion control: Controls and diagnostics for setting and measuring the pulse width are needed.

(7) NOPA gain adjustment: Techniques are needed to vary the on-target energy while maintaining the energy stability of the NOPA's that is derived from operating at saturation.

(8) Ultrabroadband front end: Although a similar prototype has been demonstrated for MTW OPAL, there remain challenges with operating such a complex system in a user facility.
These challenges are being addressed within the development of the MTW OPAL midscale laser and are described in more detail in Section 3.

\section{MTW OPAL overview}

MTW OPAL is a midscale prototype pumped by the MTW laser $^{[6,7]}$ that is being constructed to produce 7.5-J, 15-fs pulses and demonstrate technologies that are suitable for kilojoule all-OPCPA systems, such as could be pumped by OMEGA EP. In addition to being a critical platform for laser development, the MTW OPAL laser will share a target area with the existing MTW Laser System (50 J, 1 to 100 ps), making possible several joint-shot configurations.

A schematic of the laser system is shown in Figure 3. The top half shows the MTW laser with subsections highlighted that are used to pump the final stage of the OPAL, which is shown in the bottom half. The ultrabroadband front end (UFE) for the OPAL produces $600-\mu \mathrm{J}$ pulses at $5 \mathrm{~Hz}$. The pulses have $200 \mathrm{~nm}$ of bandwidth, are compressible to sub$15 \mathrm{fs}$, and are stretched to $1.5 \mathrm{~ns}$ for further amplification. The UFE, described in detail in Section 4, contains three NOPA's. Two more, NOPA4 and NOPA5, increase the energy to $12 \mathrm{~J}$ before the pulses are compressed.

Demonstrating large-aperture OPCPA using DKDP is one of the primary goals of the MTW OPAL project. DKDP is used in NOPA5 to amplify $45 \mathrm{~mm}$-square beams, and 


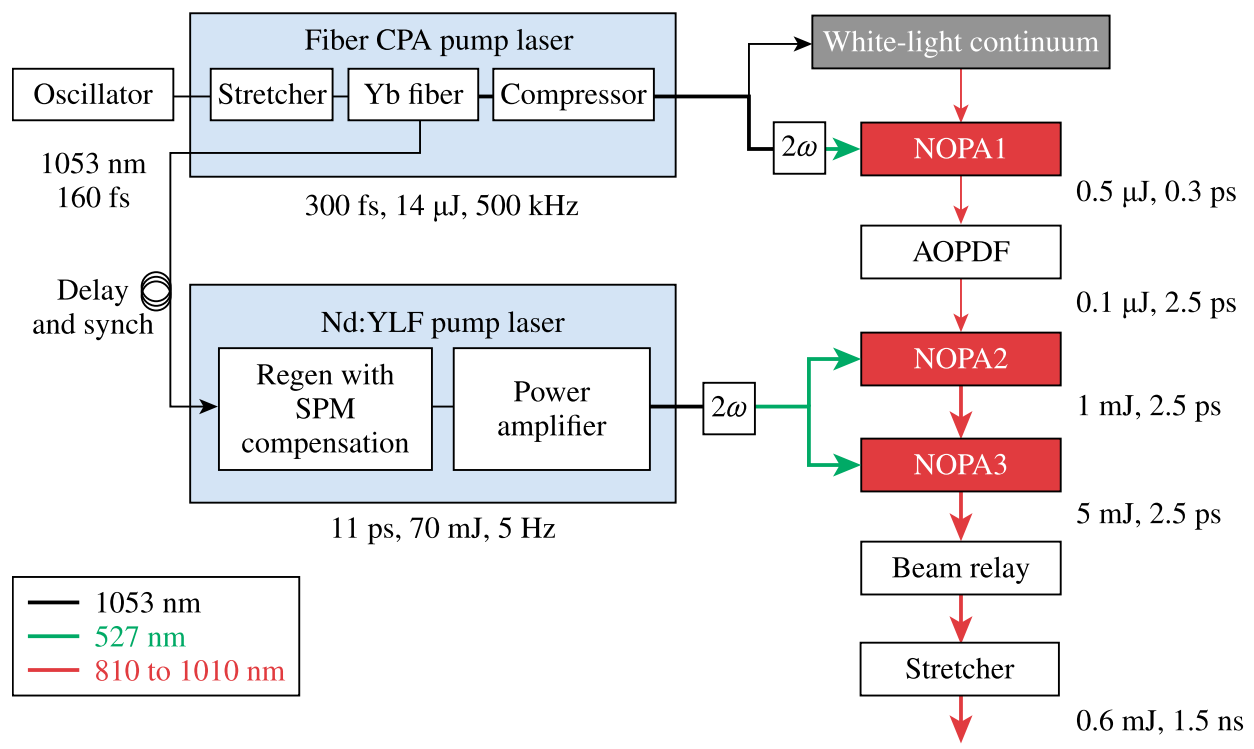

E24303J1

Figure 4. Schematic of the UFE subsystem. CPA, chirped-pulse amplification; SPM, self-phase modulation; AOPDF, acousto-optic programmable dispersive filter.

therefore the broadband spectrum of the system is centered at $\sim 910 \mathrm{~nm}$ to match the amplifier's broadband gain window. The previous amplifier stages have smaller beam sizes $(<7 \mathrm{~mm})$, and therefore $\beta$-barium borate $(\mathrm{BBO})$ is an option that is preferred because it has a higher nonlinearity coefficient ${ }^{[8]}$.

Separate pump lasers are used for NOPA4 and NOPA5, which are described in a later section. NOPA4 contains two BBO stages that are pumped using a $5-\mathrm{Hz} \mathrm{Nd}$ :YLF laser system; NOPA5 is pumped using MTW. Three switchyards (shown as circles) are used to configure MTW to provide narrowband, 80-J pump pulses at $1053 \mathrm{~nm}$. After frequency conversion, 50-J pulses at $526.5 \mathrm{~nm}$ are transported through the wall that separates the MTW and OPAL systems.

The broadband beam is image relayed between the OPAL amplifier stages using lens-based vacuum spatial filters (not shown). Longitudinal chromatic aberration in the planoconvex lenses introduces radial group delay ${ }^{[9,10]}$ that, if not corrected, would increase the duration of the compressed pulse from $<20$ fs to more than 100 fs. A number of techniques have been demonstrated to compensate for this spatiotemporal aberration ${ }^{[1-13]}$. For this system, an ultrabroadband radial group-delay compensator has been developed that uses two plano-concave lenses located before the NOPA5 amplifier in an Offner imaging system to reduce the radial group delay to $<2 \mathrm{fs}^{[14]}$. This improves the final spatiotemporal focus and eliminates phase-matching issues in NOPA5 that could arise if the beam had significant chromatic aberrations.

After NOPA5, the pulses are transported into the compressor chamber using an all-reflective, achromatic image relay (AIR) that is free of chromatic aberrations ${ }^{[15]}$. The chamber also contains a four-grating vacuum compressor, optics to provide sample beams for laser diagnostics, and optics to transport the beam to a target chamber in an experimental area that supports both MTW and OPAL for joint-shot campaigns.

\section{MTW OPAL subsystems}

Development of the MTW OPAL system is separated into subsystems, starting with the ultrabroadband front end and ending with pulse compression and transport to the target chamber. Each subsystem has a role to play in addressing the technical challenges for building an ultraintense OPCPA laser system.

\subsection{Ultrabroadband front end}

The ultrabroadband front end shown in Figure 4 consists of a mode-locked oscillator, two pump lasers, a system for generating a white-light continuum seed, three NOPA's for amplifying the seed, an acousto-optic programmable dispersive filter (AOPDF) ${ }^{[16]}$ for dispersion control, and a broadband pulse stretcher $(1.5 \mathrm{~ns} / 200 \mathrm{~nm})$.

The OPAL pulses originate as white-light continuum produced by a fiber chirped-pulse-amplification (CPA) laser system. The seed for the fiber CPA system is a modelocked oscillator (160 fs, $1053 \mathrm{~nm}, 200 \mathrm{~mW}, 76 \mathrm{MHz}$ ) that is synchronized to the Hardware Timing System that distributes timing signals throughout the MTW and OPAL Laser Systems. Pulses from the oscillator are stretched to 


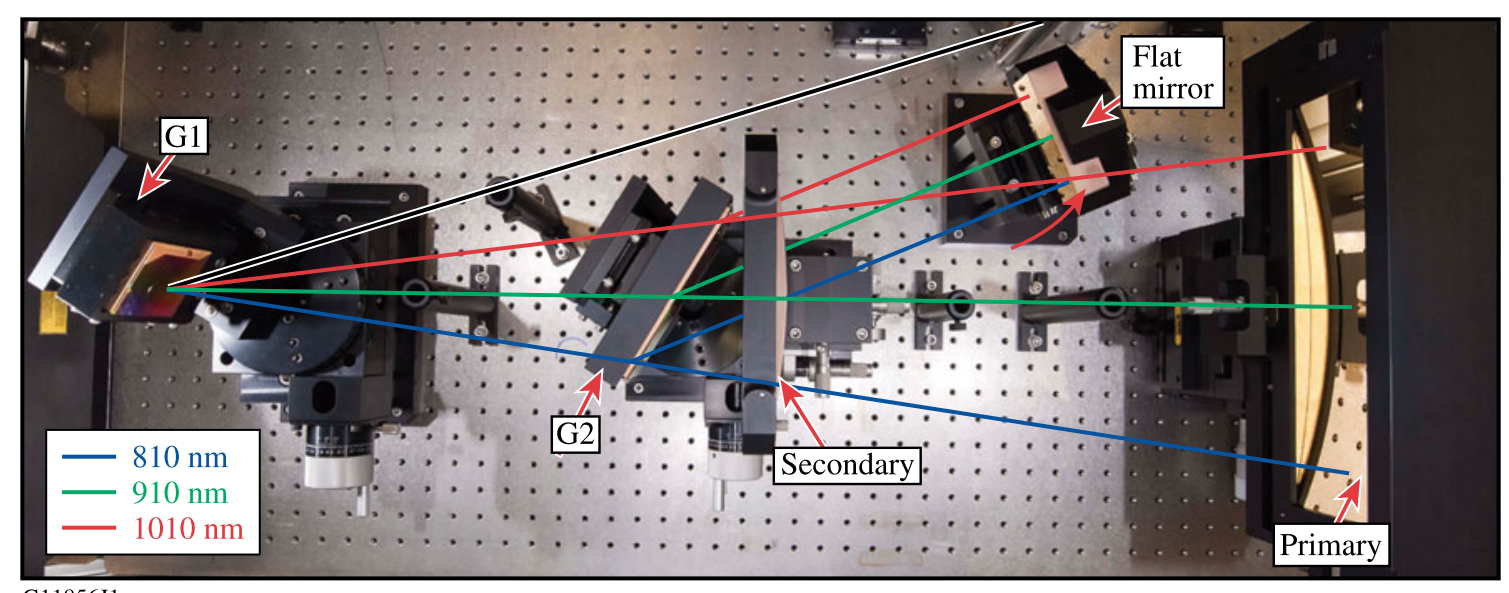

G11856J1

Figure 5. The cylindrical Offner stretcher (COS) with the single-pass beam path shown. A second pass (not shown) is achieved using a periscope oriented at $45^{\circ}$ to retroreflect the beam with a $20-\mathrm{mm}$ shift and $90^{\circ}$ beam rotation.

$180 \mathrm{ps}$ before amplification in a chain of four $\mathrm{Yb}$-doped fiber amplifiers. The final photonic crystal fiber amplifier is a double-clad design with a $55-\mu \mathrm{m}$ mode-field diameter ${ }^{[17,18]}$. Its $10-\mathrm{W}$ output at a $500-\mathrm{kHz}$ repetition rate is recompressed using multilayer dielectric gratings to produce $14-\mu \mathrm{J}, 300-\mathrm{fs}$ pulses.

The white-light continuum seed for OPAL is generated by weakly focusing a portion of the fiber CPA output $(1.6 \mu \mathrm{J})$ into a 4-mm-thick plate of yttrium aluminum garnet (YAG) $^{[19,20]}$. The remaining energy from the fiber CPA system is frequency-doubled for pumping the first optical parametric amplifier, NOPA1 in the UFE. All three BBO NOPA's have an internal noncollinear angle between the pump and signal set at $2.23^{\circ}$ for maximum bandwidth at $910 \mathrm{~nm}^{[21-23]}$.

NOPA2 and NOPA3 are pumped by a Nd:YLF pump laser that produces 11-ps, $70-\mathrm{mJ}$ pulses at $5 \mathrm{~Hz}$ that are frequencydoubled with $>60 \%$ efficiency. Picosecond pulses are used to limit temporal contrast degradation from parametric fluorescence ${ }^{[24]}$. Optical synchronization is achieved by using a portion of the pulse picked off from the fiber CPA system to seed the Nd:YLF pump laser. The diodepumped regenerative amplifier (regen) portion of the pump laser contains a BBO crystal for compensating self-phase modulation that accumulates from propagation through the cavity optics, most notably the Pockels cell ${ }^{[25,26]}$. The power amplifier contains three Nd:YLF heads that are flashlamp pumped. The pulses are frequency-doubled in lithium triborate (LBO) before they are split and image relayed to pump NOPA2 (5.5-mm thick) and NOPA3 (3.3-mm thick) with $6 \mathrm{~mJ}$ and $35 \mathrm{~mJ}$, respectively. The output energy from NOPA 3 is $5 \mathrm{~mJ}$ in a high-order, super-Gaussian round beam with $200 \mathrm{~nm}$ of bandwidth.

The dispersion of the pulses is controlled using two systems, the commercial AOPDF (DazzlerTM, HR45-650$1100)$ and a custom cylindrical Offner stretcher ${ }^{[27]}$. The
AOPDF between NOPA1 and NOPA 2 provides the ability to (a) compensate high-order dispersion at the end of the MTW OPAL system for the shortest possible pulse, and (b) stretch the pulse from $\sim 0.3 \mathrm{ps}$ to $2.5 \mathrm{ps}$ for efficient amplification in NOPA2 and NOPA3. The cylindrical Offner stretcher (COS) located after NOPA3 stretches the pulses $(1.5 \mathrm{~ns} / 200 \mathrm{~nm})$ to enable amplification in the remaining NOPA stages (see Figure 5). It uses a cylindrical Offner telescope and a pair of gratings, similar to the device demonstrated by Itatani et al. ${ }^{[28]}$, except in a double roundtrip configuration to double the dispersion and reduce the beam deformation ${ }^{[29]}$. The COS design eliminates the line focus typically found on the spherical secondary mirror of a conventional Offner stretcher. This enables pulses of higher energy to be stretched without damaging the optics. It also reduces the impact of the fine-scale surface roughness of the mirrors on the temporal contrast of the pulses ${ }^{[30]}$. A lensbased beam relay system is used to project the near-field of NOPA3 through the COS to the next NOPA stages.

Figure 6 shows typical output from the UFE. The far-field after the stretcher is symmetric with negligible power outside of the diffraction-limited spot. The spectrum is $210-\mathrm{nm}$ wide at the $10 \%$ point and has a shot-to-shot stability of $<5 \%$ (rms) across that range. The modulation is caused by parasitic processes in NOPA $1^{[31]}$, which must be operated in a walk-off-compensated geometry because of the small beam sizes $(\sim 100-\mu \mathrm{m}$ full width at half-maximum (FWHM)). The beam size in all following amplifiers (NOPA2-NOPA5) is large enough $(>2 \mathrm{~mm}$ ) such that walk-off is not detrimental, and therefore one can use a non-walk-off-compensated configuration without parasitic second-harmonic generation of the signal and idler ${ }^{[31]}$. The temporal intensity contrast of the front end was measured after NOPA1 to have an upper bound of $10^{-12}$ set by the diagnostic noise floor ${ }^{[32]}$, which is shown as the gray line. The red line indicates the upper bound after further amplification in the NOPA2 and NOPA3, which 
(a) Stretcher near field

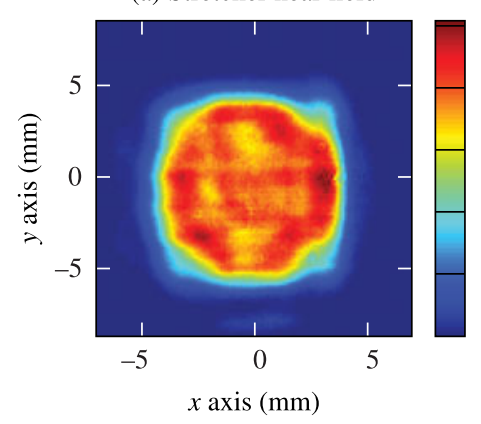

(c) Stretcher output spectrum

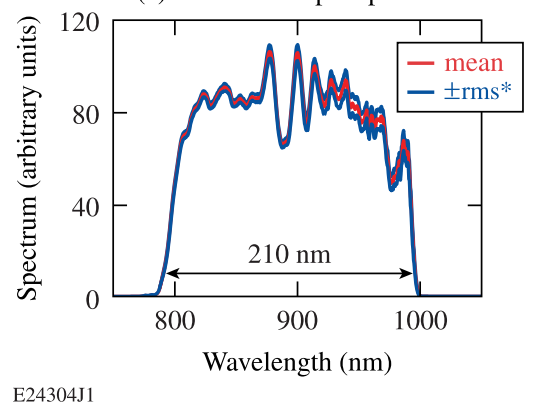

(b) Stretcher far field

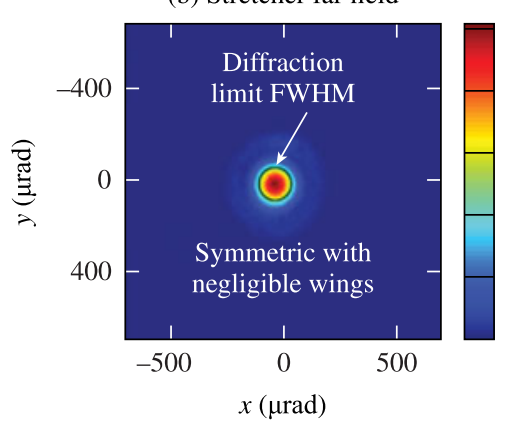

(d) Prestretch temporal contrast

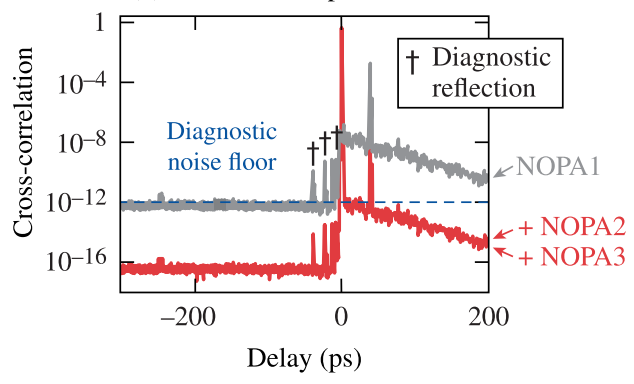

Figure 6. Stretcher output: (a) near-field, (b) far-field, and (c) spectrum. (d) Temporal contrast measured after NOPA1 and predicted for NOPA2 and NOPA3.

is temporally confined by the duration of their picosecond pump pulses ${ }^{[24]}$.

\subsection{NOPA4 amplifier stages}

NOPA4 consists of two separate BBO crystals - a preamplifier and power amplifier. They are pumped using a Nd:YLF laser that is electronically synchronized with the UFE and the MTW laser, which pumps the final stage (NOPA5). The NOPA4 pump laser is very similar to those used to pump the OPCPA stages of OMEGA EP and it operates at $5 \mathrm{~Hz}^{[33]}$.

For maximum OPCPA efficiency, the pump pulses are shaped as high-order super-Gaussians to be flat in space and time. The fiber front end for the pump laser contains an arbitrary waveform generator for shaping a 1.5-ns pulse to precompensate for gain saturation within the laser. A diode-pumped Nd:YLF regenerative amplifier, similar to the one in the ultrabroadband front end, amplifies the pulses from hundreds of picojoules to a few millijoules. The output Gaussian beam is apodized to a square profile before it is injected into a multipass crystal large-aperture ring amplifier that uses two 25-mm-diam Nd:YLF flash-lamppumped heads in a ring geometry to increase the pulse energy to $\sim 2 \mathrm{~J}$.

The pump pulses are frequency-doubled in LBO (8-mm thick) with $70 \%$ conversion efficiency before being split and image relayed to two crystals, NOPA4a (10-mm thick) and NOPA4b (4-mm thick). The first is a preamplifier that increases the energy of the stretched pulses from $300 \mu \mathrm{J}$ to
$25 \mathrm{~mJ}$. The second is a power amplifier that increases the energy to $>250 \mathrm{~mJ}$. The pump-pulse energies for the stages are $0.1 \mathrm{~J}$ and $1.0 \mathrm{~J}$, respectively. The pump beams are independently vacuum image relayed to each stage with magnification suitable to maintain a pump intensity of $2 \mathrm{GW} / \mathrm{cm}^{2}$ in a 1.5-ns pulse. The signal beam is upcollimated after NOPA4a with a vacuum image relay with a magnification of 3.3 to match the pump spatially and temporally at NOPA $4 \mathrm{~b}$. This portion of the system is being activated.

\subsection{Diagnostic compressor package}

The diagnostic compressor package (DCP) is being used to study recompression of the pulses from NOPA4 that have been intercepted using a switchyard (SY) mirror. The compressor within the DCP uses two gratings with $1285-1 / \mathrm{mm}$ groove densities (G1 and G2) and a roof mirror (RM) in the standard double-pass configuration (see Figure 7). Goldcoated, holographic gratings, identical to those required for the final vacuum compressor, are used to compress pulses to less than $20 \mathrm{fs}$, which are measured using SPIDER ${ }^{[34,35]}$.

The DCP beam is $\sim 40 \%$ the size of the full-energy beam from NOPA5 that will be sent to the four-grating vacuum compressor. Therefore, the DCP can use the doublepass, two-grating configuration, which is more compact and less expensive. However, the $35-\mathrm{mm}$-square beam is large enough so that $10-\mathrm{mJ}$ compressed pulses can be passed into a vacuum chamber for femtosecond laser-damage testing without accumulating an unacceptable amount of self-phase modulation from propagation through the vacuum window. 


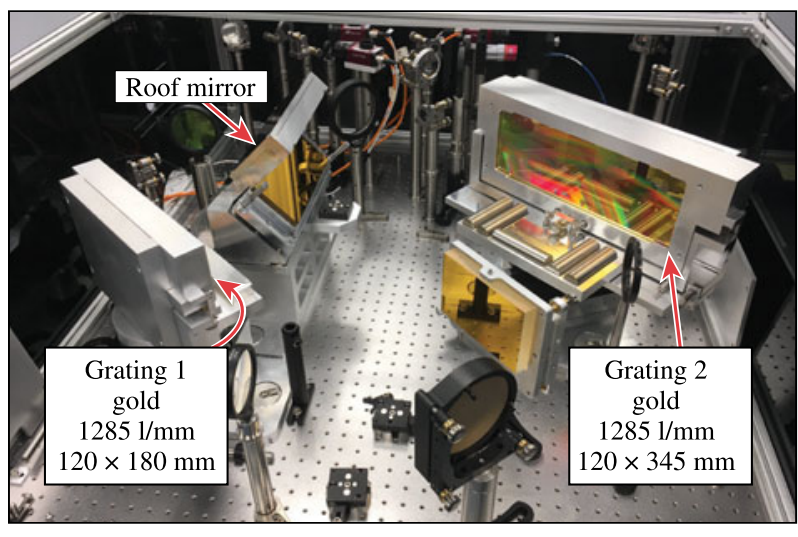

G11858J1

Figure 7. Photo of the diagnostic compressor package (DCP), showing the two gratings, mount, and the roof mirror that is used for the two-pass configuration.

Five of the eight technical challenges are being addressed with the DCP subsystem: developing gratings (\#1), coatings (\#4), diagnostics (\#5), demonstrating dispersion control (\#6), and validating the performance of the UFE (\#8). The DCP is being used to test the full-size gold gratings required for the vacuum compressor, and the precision optical mounts and alignment techniques necessary for compressing the pulses from NOPA4 to $\sim 15$ fs. The output from the DCP will be used for femtosecond laser-damage testing in vacuum - a capability that is critical for supporting the development of the ultrabroadband gratings and the coatings required in the vacuum compressor. The DCP is advancing the development of short-pulse diagnostics by providing a test bed for measuring compressed pulses with enough energy for a high-dynamic-range contrast measurement using a commercial third-order cross-correlator, Sequoia ${ }^{\mathrm{TM}}{ }^{36]}$. These diagnostics will, in turn, enable validation of the UFE. Specifically, they will be used to test the performance of the AOPDF and COS for dispersion control.

Initial measurements have shown that the AOPDF in the UFE is effective for compensating higher-order dispersion, as intended, to achieve sub-20 fs pulses. Figure 8(a) shows two spectral phase measurements made with a SPIDER device after the DCP with pulses from UFE. The initial spectral phase (blue curve) was subtracted from the AOPDF dispersion using the Dazzler software, resulting in the second measurement (red curve). Figure 8(b) shows the improvement in the temporal pulse that was calculated using the super-Gaussian spectrum that is expected from NOPA4 after activation (820 to $1000 \mathrm{~nm}$ FWHM).

\subsection{Midscale noncollinear optical parametric amplifier (NOPA5)}

NOPA5 is the last noncollinear optical parametric amplifier stage in the MTW OPAL system. It is pumped using 1.5-ns (a)
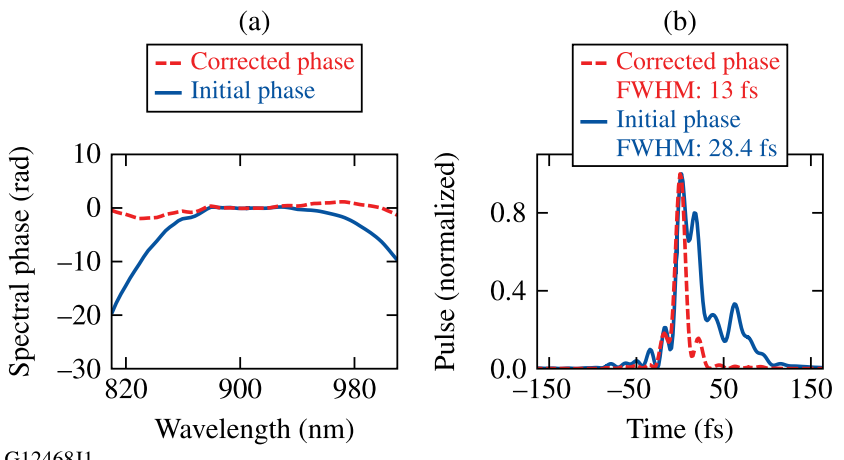

Figure 8. (a) SPIDER measurements of the spectral phase of the UFE pulses after compression in the DCP, before and after correction using the AOPDF. (b) Corresponding temporal pulse shapes calculated using the measured spectral phases and the super-Gaussian spectrum expected from NOPA4 after activation.

pulses at $527 \mathrm{~nm}$ from MTW, when operated in a narrowband mode, where the OPCPA portion of MTW is bypassed as shown in Figure 3. MTW can be fired once every $20 \mathrm{~min}$, set by the cool-down time of the 150-mm-aperture, Nd:glass disk amplifier, which is used in a four-pass configuration ${ }^{[6]}$. Adaptive beam shaping with a programmable liquid crystal spatial light modulator is used to control the beam size and super-Gaussian order at the NOPA5 crystal $^{[37]}$.

The $60 \mathrm{~mm}$, clear-aperture requirement for the NOPA5 crystal means that the crystal type used in the preceding NOPA stages (BBO) cannot be used because of commercial availability. Only DKDP can provide the required bandwidth over this aperture size. Simulations of NOPA5 predict amplified pulses with $>180 \mathrm{~nm}$ of bandwidth ( 830 to $1010 \mathrm{~nm}$ ), and energies exceeding $12.5 \mathrm{~J}$ when pumped with $50 \mathrm{~J}$. Optimum values of the noncollinear angle between the pump and signal $\mathbf{k}$ vectors $\left(\sim 0.9^{\circ}\right.$ internal) and deuteration levels $(\sim 90 \%)$ are being analyzed numerically and experimentally.

Figure 9 shows the two periscope assemblies that use dichroic mirrors to combine the pump and seed beams and separate residual pump light after the amplifier. The broadband signal beam is vertically polarized and passes through the lower mirror at Brewster's angle. The pump is horizontally polarized and the crystal is rotated about a vertical axis for Type-I phase matching. An external noncollinear angle of $\sim 1.3^{\circ}$ (corresponding to an internal angle of $\sim 0.9^{\circ}$ ) is required between the pump and signal in the horizontal plane to obtain broadband phase matching. The specific angles for broadest gain and phase matching depend on the DKDP deuteration level.

An angularly dispersed idler beam, produced in NOPA5 to conserve energy and momentum, propagates from the NOPA5 crystal at a nominal $3^{\circ}$ angle to the signal beam. The wavelength range of the idler is $1102-1443 \mathrm{~nm}$, corresponding to the signal range of $830-1010 \mathrm{~nm}$ and the pump wavelength of $527 \mathrm{~nm}$. The idler beam passes through the 


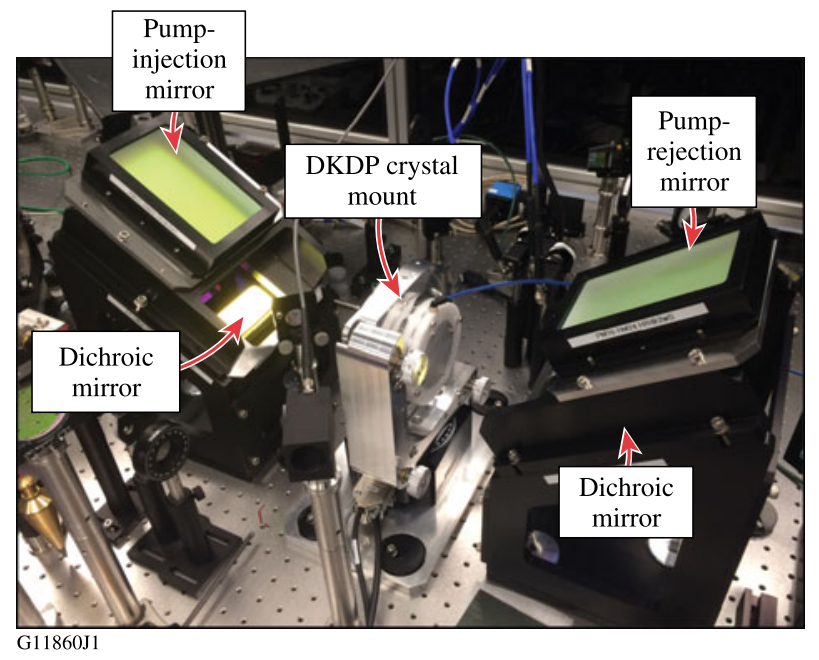

Figure 9. The NOPA5 amplifier showing the two dichroic periscope mirror assemblies that are used to combine the pump and seed beams and separate any residual pump from the amplified signal.

output dichroic mirror but it is blocked by a custom shortwavelength-pass filter that was designed and produced at LLE for this project. Additional idler rejection is achieved using a large iris in the far-field plane of the following achromatic image relay and by the low diffraction efficiency of the gratings at the idler wavelengths.

NOPA5 development addresses technical challenge \#3, obtaining crystals of highly deuterated DKDP $(\sim 90 \%)$ with sufficient deuteration uniformity $(<1 \%$ variation across the aperture) and optical quality. Currently, a set of crystals of different deuteration levels ranging from $80 \%$ to $98 \%$ are being tested, which have smaller apertures $(25 \mathrm{~mm}$ square) than the full-scale crystal (60 $\mathrm{mm}$ square), but the same nominal 48-mm length. Results from these tests are being used to refine the NOPA5 simulations before fullsize crystals at the optimum deuteration level and length are purchased.

Figure 10 shows a two-wavelength tuning-curve setup that is being used to test models of NOPA5. The beam from two cw fiber-coupled laser diodes at 850 and $976 \mathrm{~nm}$ is collimated and aligned precisely through the sub-aperture NOPA5 crystals with a noncollinear angle, $\alpha_{\text {external }}$, to the MTW pump beam. Amplification of the seed beam at these two wavelengths is measured as a function of the phase-matching angle using a photodiode and a $12-\mathrm{GHz}$ oscilloscope after separation using a diffraction grating. Measurements shown for one crystal $(D=92 \%)$, show that increasing $\alpha_{\text {external }}$ from $1.15^{\circ}$ to $1.35^{\circ}$ improved the overlap of the two curves, corresponding to a higherbandwidth alignment configuration between the pump, seed, and crystal.

In addition to testing DKDP crystals, NOPA5 will be used to test beam energy adjustment (\#7), using the programmable pulse and beam shaping capabilities of the MTW pump laser,

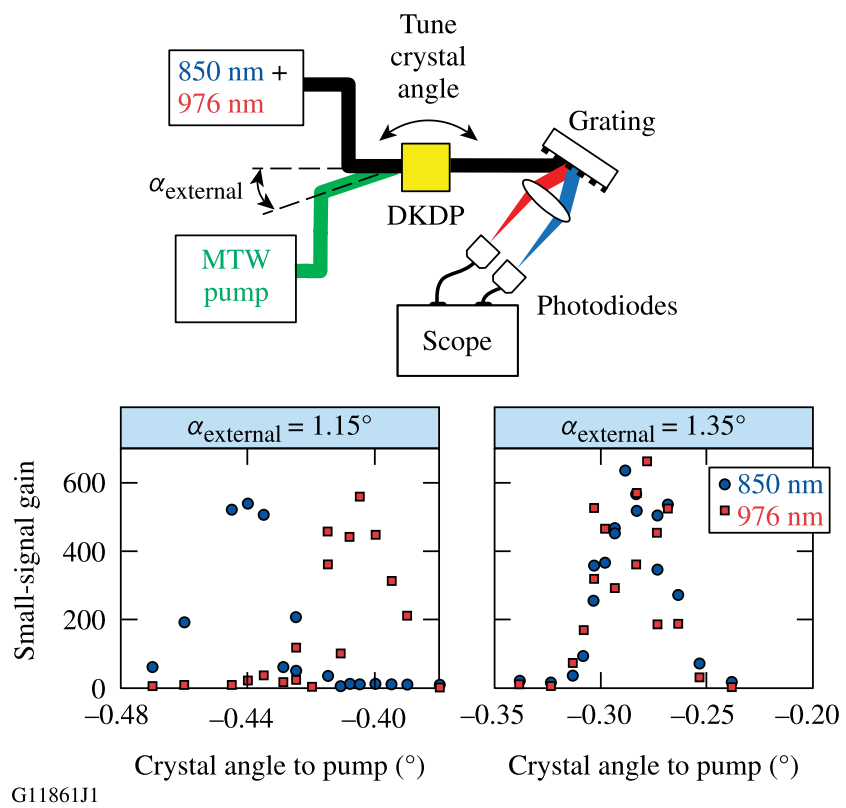

Figure 10. Schematic of the two-wavelength tuning-curve setup that is being used to determine the optimum noncollinear angle, $\alpha_{\text {external, }}$, for broadband gain.

and to test full-aperture beam shapers that will be placed immediately after NOPA5 to control the beam profile before propagation into the compressor chamber.

\subsection{Ultrabroadband transport}

Ultrabroadband transport refers not to a specific system, but rather the components and strategies required to propagate pulses from the start of the UFE, through the amplifier stages via the image relay optics, into the vacuum compressor chamber, and on to the focal spot at the target location. The portion of the system up to NOPA5 has been completed and is being tested. The remaining subsystems are being designed and portions will be tested offline before final installation.

One challenge the transport system will address is ultrabroadband wavefront control and focusing (\#2). The radial group-delay compensator (RGDC) uses an all-reflective spherical Offner triplet to image the beam through two plano-concave lenses to cancel longitudinal chromatic aberration that is introduced by the lens-based image relays between the amplifier stages ${ }^{[14]}$. A compact, spatially resolved spectral interferometry technique was developed to test this approach $^{[38]}$. After amplification in NOPA5, the beam will be apodized and propagate through a window into a large vacuum system that will contain all subsequent optics. An AIR will use four spherical mirrors in a low-aberration configuration to image relay NOPA5 to the vacuum compressor with a $2 \times$ beam expansion. An offline AIR test bed has been 


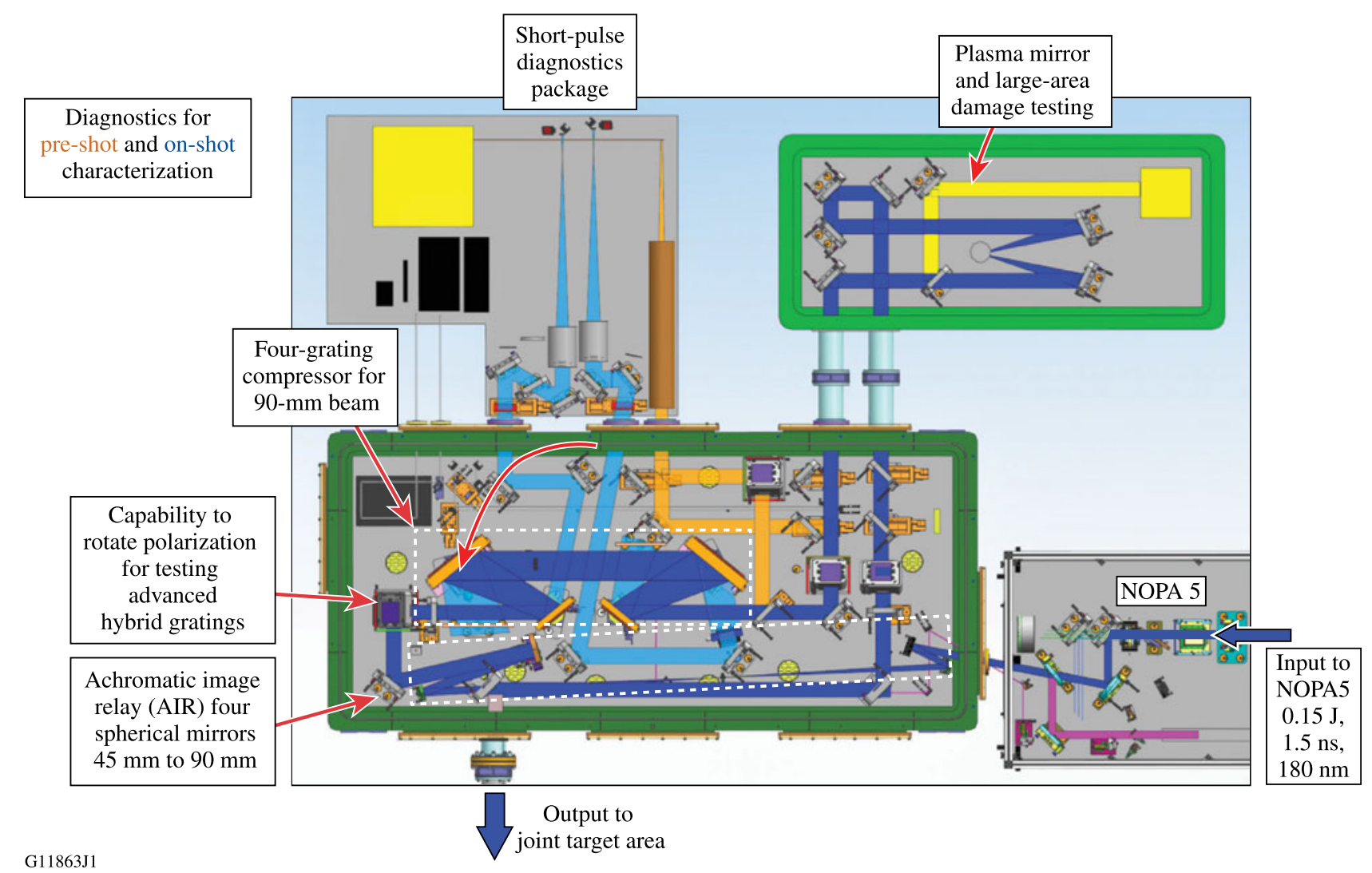

Figure 11. Schematic of the grating compressor chamber showing the major subsystems and main beam path (dark blue), pre-shot, low-energy beam path for shot setup (orange), and the diagnostic beam paths used for on-shot diagnostics (light blue).

built to develop alignment techniques and qualify the optics before they are installed in the vacuum chamber.

The ultrabroadband transport system is also addressing the challenge of developing short-pulse coatings (\#4). Before the vacuum compressor, broadband mirrors are required for s-polarized, 1.5-ns pulses, propagating in air and vacuum with fluences up to $\sim 1 \mathrm{~J} / \mathrm{cm}^{2}$, and for which dielectric mirrors have been developed. After the compressor, mirrors capable of transporting 20-fs pulses in vacuum with nominal fluences of $100 \mathrm{~mJ} / \mathrm{cm}^{2}$ are required, suitable for both $\mathrm{s}$ and p polarizations. Small-scale prototypes for all of the required coatings have been manufactured and tested at $800 \mathrm{~nm}^{[39]}$. The remaining development includes optimizing the designs after measuring the femtosecond damage thresholds at 920 $\mathrm{nm}$ using the femtosecond damage test station. Finally, fullsize mirrors for the $90 \mathrm{~mm}$-square beam will be coated in an LLE e-beam coating chamber that was designed to have a layer uniformity of $<0.1 \%$ over the 300 -mm coating area ${ }^{[39]}$.

\subsection{Vacuum compressor}

The vacuum compressor will be used to demonstrate fullenergy dispersion compensation at the end of the system by compressing the 1.5 -ns pulses from NOPA 5 to $<20 \mathrm{fs}$; there- fore, it plays a key role in addressing technical challenge \#6 (ultrabroadband dispersion control). Figure 11 shows this portion of the system from the input from NOPA5, the AIR, the grating compressor itself, the short-pulse diagnostics package, a separate plasma mirror chamber, and the output to the target chamber.

The compressor uses a four-grating configuration, consisting of two pairs of parallel gratings, that is better suited to the $90 \mathrm{~mm}$-square beam than the two-grating design of the DCP that requires a roof mirror. Right-angle periscopes before and after the compressor will be used to rotate the beam. This is necessary because gold gratings, the only type currently commercially available, are optimized for input beams that are p polarized. This type of grating is etched into a dielectric substrate that is then overcoated with gold to increase the reflectivity over the bandwidth.

Advanced 'hybrid' gratings, consisting of a dielectric grating that is written on top of a metal reflective layer, are being developed with the goal of increasing the damage threshold while providing sufficient bandwidth for sub-20 fs pulses $^{[40,41]}$. The concept is that the all-dielectric grating diffracts a significant portion of the laser power, shielding the lower-damage-threshold gold layer that provides the bandwidth. Unlike standard gold gratings, advanced gratings require s-polarized beams. To accommodate this, should 


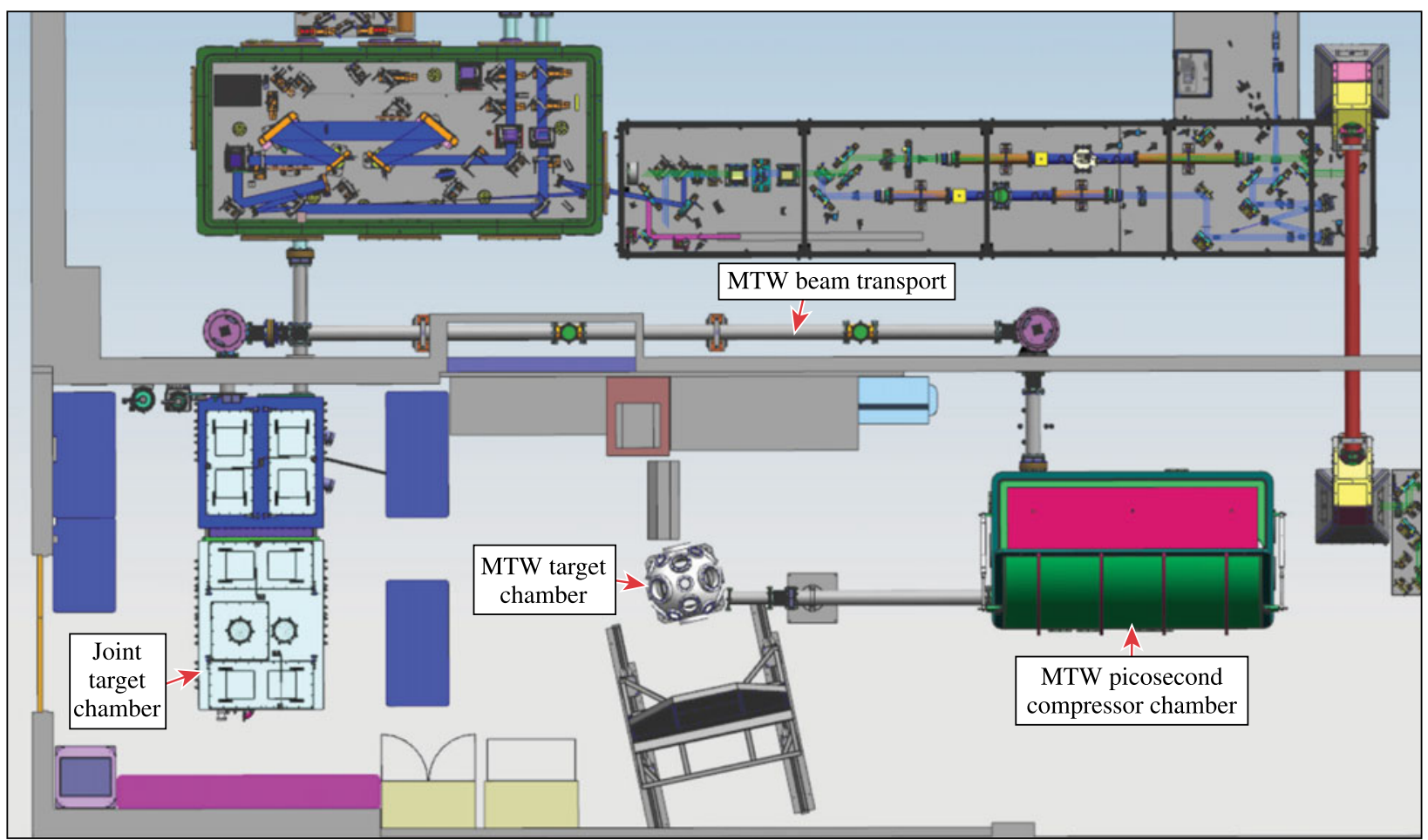

G11866J1

Figure 12. Schematic of the back end of the laser systems.

such advanced gratings become available at the required sizes $(120 \times 345 \mathrm{~mm})$, the beam transport before and after the vacuum compressor will permit in-line periscopes and turning mirrors to be used (instead of the right-angle periscopes) to preserve the input s-polarization state through the compressor.

\subsection{Plasma optics and target science}

The portion of the system after the compressor will be used to address the technical challenges of focusing ultrabroadband petawatt-class pulses (\#2). After compression, a pair of switchyard mirrors will set the beam path. One option will be to send the beam directly to the target chamber where it will be focused on the target using an off-axis parabola, with or without a refocusing ellipsoidal mirror. Alternatively, it will first be switched through a plasma mirror chamber, which will be differentially pumped to protect the compressor gratings from contamination, and will contain a pair of plasma mirrors for improving the temporal contrast ${ }^{[42]}$. The plasma mirror chamber will also include a site for large-area damage testing and for testing other types of plasma mirrors in refocusing geometries.

Figure 12 shows the overall layout of both back ends for the picosecond system (MTW) and femtosecond system (MTW OPAL). Either laser will be available for single-shot experiments at full energy in the joint target chamber. In addition, MTW OPAL will be available at reduced energy ( $100 \mathrm{~mJ}$ with NOPA4 only) for joint experiments with MTW in either nanosecond or picosecond mode. The long axis of the joint target chamber enables a range of $f$ /\#'s $(\sim 1$ to 20) to be used for different physics experiments.

\section{Conclusions}

The development of a midscale laser system, MTW OPAL, is nearing completion to demonstrate laser technologies suitable for ultraintense, all-OPCPA systems. Although the MTW OPAL specification of $0.5 \mathrm{PW}$ is modest compared to the best Ti:sapphire systems, the OPCPA platform consisting of DKDP crystals pumped by $\mathrm{Nd}$ :glass lasers is scalable in principle to much higher powers for sub-20 fs pulses with kilojoule energies, without limitations from transverse amplified spontaneous emission or gain for back-reflected light. MTW OPAL is a critical stepping stone for technology development and for making a credible proposal for a fullscale 75-PW system.

\section{Acknowledgements}

This material is based upon work supported by the Department of Energy, National Nuclear Security Administration under Award Number DE-NA0001944, the University 
of Rochester, and the New York State Energy Research and Development Authority. This report was prepared as an account of work sponsored by an agency of the U.S. Government. Neither the U.S. Government nor any agency thereof, nor any of their employees, makes any warranty, express or implied, or assumes any legal liability or responsibility for the accuracy, completeness, or usefulness of any information, apparatus, product, or process disclosed, or represents that its use would not infringe privately owned rights. Reference herein to any specific commercial product, process, or service by trade name, trademark, manufacturer, or otherwise does not necessarily constitute or imply its endorsement, recommendation, or favoring by the U.S. Government or any agency thereof. The views and opinions of authors expressed herein do not necessarily state or reflect those of the U.S. Government or any agency thereof.

\section{References}

1. I. N. Ross, P. Matousek, M. Towrie, A. J. Langley, and J. L. Collier, Opt. Commun. 144, 125 (1997).

2. V. V. Lozhkarev, G. I. Freidman, V. N. Ginzburg, E. A. Khazanov, O. V. Palashov, A. M. Sergeev, and I. V. Yakovlev, Laser Phys. 15, 1319 (2005).

3. Y. Tang, I. N. Ross, C. Hernandez-Gomez, G. H. C. New, I. Musgrave, O. V. Chekhlov, P. Matousek, and J. L. Collier, Opt. Lett. 33, 2386 (2008).

4. J. H. Kelly, L. J. Waxer, V. Bagnoud, I. A. Begishev, J. Bromage, B. E. Kruschwitz, T. J. Kessler, S. J. Loucks, D. N. Maywar, R. L. Mccrory, D. D. Meyerhofer, S. F. B. Morse, J. B. Oliver, A. L. Rigatti, A. W. Schmid, C. Stoeckl, S. Dalton, L. Folnsbee, M. J. Guardalben, R. Jungquist, J. Puth, M. J. Shoup, III, D. Weiner, and J. D. Zuegel, J. Phys. IV 133, 75 (2006).

5. A. Kon, M. Nakatsutsumi, S. Buffechoux, Z. L. Chen, J. Fuchs, Z. Jin, and R. Kodama, J. Phys.: Conf. Ser. 244, 032008 (2010).

6. V. Bagnoud, J. Puth, I. Begishev, M. Guardalben, J. D. Zuegel, N. Forget, and C. Le Blanc, in Conference on Lasers and Electro-Optics/Quantum Electronics and Laser Science and Photonic Applications, Systems and Technologies, Technical Digest (CD) (Optical Society of America, 2005), paper JFA1.

7. I. A. Begishev, J. Bromage, S.-W. Bahk, R. Cuffney, C. Dorrer, D. Haberberger, D. H. Froula, C. Mileham, P. M. Nilson, C. Stoeckl, and J. D. Zuegel, in 7th International Conference on Ultrahigh Intensity Lasers, Montebello, Quebec, Canada, 1116 September (2016).

8. V. G. Dmitriev, G. G. Gurzadyan, and D. N. Nikogosyan, Handbook of Nonlinear Optical Crystals (Springer, Berlin, 1991), p. 78

9. Z. Bor, J. Mod. Opt. 35, 1907 (1988).

10. H.-M. Heuck, P. Neumayer, T. Kühl, and U. Wittrock, Appl. Phys. B 84, 421 (2006).

11. T. J. Kessler, H. Huang, and D. Weiner, in International Conference on Ultrahigh Intensity Laser Development, Science and Emerging Applications 2006 (ICUIL 2006) (2006), p. 126.

12. J. Néauport, N. Blanchot, C. Rouyer, and C. Sauteret, Appl. Opt. 46, 1568 (2007).
13. E. W. Gaul, M. Martinez, J. Blakeney, A. Jochmann, M. Ringuette, D. Hammond, T. Borger, R. Escamilla, S. Douglas, W. Henderson, G. Dyer, A. Erlandson, R. Cross, J. Caird, C. Ebbers, and T. Ditmire, Appl. Opt. 49, 1676 (2010).

14. S.-W. Bahk, J. Bromage, and J. D. Zuegel, Opt. Lett. 39, 1081 (2014).

15. E. M. Schiesser, S.-W. Bahk, and P. Rolland, in Optical Design and Fabrication 2017 (Optical Society of America, 2017), paper JTu3A.6.

16. P. Tournois, Opt. Commun. 140, 245 (1997).

17. O. Schmidt, J. Rothhardt, T. Eidam, F. Röser, J. Limpert, A. Tünnermann, K. P. Hansen, C. Jakobsen, and J. Broeng, Opt. Express 16, 3918 (2008).

18. J. Bromage, J. M. Fini, C. Dorrer, and J. D. Zuegel, Appl. Opt. 50, 2001 (2011).

19. M. Bradler, P. Baum, and E. Riedle, Appl. Phys. B 97, 561 (2009).

20. R. L. Fork, C. V. Shank, C. Hirlimann, R. Yen, and W. J. Tomlinson, Opt. Lett. 8, 1 (1983).

21. G. Cerullo and S. De Silvestri, Rev. Sci. Instrum. 74, 1 (2003).

22. G. M. Gale, M. Cavallari, T. J. Driscoll, and F. Hache, Opt. Lett. 20, 1562 (1995).

23. D. N. Schimpf, J. Rothhardt, J. Limpert, A. Tünnermann, and D. C. Hanna, J. Opt. Soc. Am. B 24, 2837 (2007).

24. C. Dorrer, A. V. Okishev, and J. D. Zuegel, Opt. Lett. 32, 2143 (2007).

25. C. Dorrer, R. G. Roides, J. Bromage, and J. D. Zuegel, Opt. Lett. 39, 4466 (2014)

26. C. Dorrer, J. Opt. Soc. Am. B 31, 1891 (2014).

27. J. Bromage, M. Millecchia, J. Bunkenburg, R. K. Jungquist, C. Dorrer, and J. D. Zuegel, in Ultrafast Optics 2011, Monterey, CA, USA, 26-30 September (2011).

28. J. Itatani, Y. Nabekawa, K. Kondo, and S. Watanabe, Opt. Commun. 134, 134 (1997).

29. J. Bromage, M. Millecchia, J. Bunkenburg, R. K. Jungquist, C. Dorrer, and J. D. Zuegel, in Conference on Lasers and ElectroOptics (Optical Society of America, 2012), paper CM4D.4.

30. J. Bromage, C. Dorrer, and R. K. Jungquist, J. Opt. Soc. Am. B 29, 1125 (2012).

31. J. Bromage, J. Rothhardt, S. Hädrich, C. Dorrer, C. Jocher, S. Demmler, J. Limpert, A. Tünnermann, and J. D. Zuegel, Opt. Express 19, 16797 (2011).

32. J. Bromage, C. Dorrer, and J. D. Zuegel, J. Opt. 17, 094006 (2015).

33. V. Bagnoud, M. J. Guardalben, J. Puth, J. D. Zuegel, T. Mooney, and P. Dumas, Appl. Opt. 44, 282 (2005).

34. C. Dorrer, B. de Beauvoir, C. Le Blanc, S. Ranc, J.-P. Rousseau, P. Rousseau, and J.-P. Chambaret, Opt. Lett. 24, 1644 (1999)

35. C. Iaconis and I. A. Walmsley, Opt. Lett. 23, 792 (1998).

36. http://www.amplitude-technologies.com/.

37. S.-W. Bahk, I. A. Begishev, and J. D. Zuegel, Opt. Commun. 333, 45 (2014).

38. S.-W. Bahk, C. Dorrer, R. G. Roides, and J. Bromage, Appl. Opt. 55, 2413 (2016).

39. J. B. Oliver, J. Bromage, C. Smith, D. Sadowski, C. Dorrer, and A. L. Rigatti, Appl. Opt. 53, A221 (2014).

40. F. Canova, O. Uteza, J.-P. Chambaret, M. Flury, S. Tonchev, R. Fechner, and O. Parriaux, Opt. Express 15, 15324 (2007).

41. J. Wang, Y. Jin, J. Ma, T. Sun, and X. Jing, Appl. Opt. 49, 2969 (2010).

42. A. Lévy, T. Ceccotti, P. D’Oliveira, F. Réau, M. Perdrix, F. Quéré, P. Monot, M. Bougeard, H. Lagadec, P. Martin, J.-P. Geindre, and P. Audebert, Opt. Lett. 32, 310 (2007). 